\title{
Unifying cancer genetics
}

\author{
Sharon E. Plon, $M D, P h D$
}

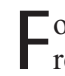
or the past several decades, the field of cancer genetics has really been two fields. Medical geneticists and genetic counselors use the term to describe inherited susceptibility to cancer and the identification of constitutional mutations, which convey this risk. Alternatively, many pathologists, oncologists, and molecular biologists use the term to describe the multitude of genetic changes that occur in the tumor cell itself. Even among the American Board of Medical Genetics laboratory specialties, molecular diagnosticians have focused on inherited mutations, e.g., sequencing DNA from blood for tumor suppressor gene mutations, whereas cytogeneticists and molecular pathologists characterize translocations, copy number changes, and specific oncogenic missense mutations in tumor specimens.

This dichotomy is breaking down. Like most things in cancer genetics, one can always start with retinoblastoma. The determination as to whether a patient with unilateral retinoblastoma has the hereditary form is founded on analysis of the tumor specimen to identify both $R B 1$ inactivating events. ${ }^{1}$ This information is then used to inform analysis of the blood, e.g., if the $R B 1$ promoter undergoes biallelic methylation, then analysis of the blood is not indicated. The wealth of data from the Cancer Genome Anatomy project (TCGA) analysis of glioblastoma reveals that somatic mutations in susceptibility genes like $R B 1$ are more frequent than first realized. ${ }^{2}$ As next generation sequencing becomes increasingly available, practitioners of all types will need to consider both the constitutional and cancer genome when making clinical decisions. Described here are some examples of how medical geneticists are beginning to incorporate cancer genome data into clinical practice.

Analysis of relatively rare gastrointestinal tumors demonstrates the interaction between genes in the same signaling pathway. Molecular pathologists have demonstrated that a substantial portion of both hepatoblastoma and desmoid tumors contain mutations that impact WNT signaling. ${ }^{3}$ In the majority of cases, these mutations are specific exon 3 missense mutations in the $C T N N B 1$ gene encoding $\beta$-CATENIN. ${ }^{4}$ In contrast, work on hereditary tumors has demonstrated that a subset of patients with these tumors (perhaps $10-15 \%$ ) harbor germline mutations in the $A P C$ gene even if other clinical features of familial adenomatous polyposis are not yet evident. ${ }^{5}$ Thus, diagnostic sequencing of the blood for mutations in $A P C$ has been recommended for all children with these tumors. ${ }^{5}$ APC and $\beta$-CATENIN proteins function in the same pathway where APC regulates the availability of $\beta$-CATENIN. More recently, an inverse relationship between somatic CTNNB1 mutation and constitutional $A P C$ mutation in desmoid and hepatoblastoma tumors has been established. ${ }^{4,6}$ If the tumor contains a somatic mutation in CTNNB1, then constitutional APC mutations are not found. So now when I am referred a child with one of these tumors,

From the Texas Children's Cancer Center, Baylor College of Medicine, Houston, Texas.

Sharon E. Plon, MD, PhD, Feigin Center Room 1200.18, 1102 Bates Street, Houston, TX 77030. E-mail: splon@bcm.tmc.edu.

Disclosure: The author declares no conflict of interest

Published online ahead of print February 9, 2011.

DOI: $10.1097 /$ GIM.0b013e31820d5e87 my first step is to find what the molecular pathology of the tumor showed. If one of the recurrent CTNNB1 missense somatic mutations is found, then the much more expensive comprehensive analysis of $A P C$ from the blood is generally not indicated. There are many other examples of a given tumor type requiring disruption of only one gene in a pathway. Juvenile myelomonocytic leukemia can result from inheriting a mutation in the $N F 1$ or $C B L$ gene or somatic mutation of the Ras signaling pathway including mutations in the PTPN11, NRAS, KRAS, or ASXL1 genes (Fig. 1). ${ }^{7}$ Large-scale genome analysis of glioblastomas demonstrate that for a given signaling pathway, brain tumors contain a variety of different mutational hits but any given tumor contains on average disruption of one gene in the pathway. ${ }^{2}$ Thus, we need to think about analysis of key signaling pathways involved in tumor development and distinguish when the initial lesion is an inherited mutation or results from a somatic event.

Colon cancer is one of the best examples of where population-based molecular analysis of all tumors has been initiated by geneticists, oncologists, gastroenterologists, and pathologists based on knowledge of Lynch syndrome (hereditary nonpolyposis colon cancer) and the microsatellite instability (MIN) that results from defects in mismatch repair genes. The presence of MIN or the lack of expression of one of the proteins detected by immunohistochemistry is used to decide upon further molecular analyses and whether germline testing is required. ${ }^{8}$ But colon cancer also demonstrates inverse relationships between germline and somatic mutations that can not be easily understood by simple pathway analysis. Approximately, $40 \%$ of MIN tumors harbor specific missense mutations in the $B R A F$ oncogene. ${ }^{9,10}$ For reasons that are not clearly understood, tumors with somatic $B R A F$ mutations almost never derive from patients with Lynch syndrome. $B R A F$ mutations are seen in tumors with somatic methylation of $M L H 1$ and the $B R A F$ mutations may be an early event that is associated with abnormal methylation of multiple genes in the tumor. ${ }^{11}$ Therefore, current recommendations for molecular diagnosis of colon cancer include MIN or immunohistochemistry analysis combined with $B R A F$ mutation status to decide whether susceptibility testing is indicated. Thus, the results of the tumor analysis impact prognosis and treatment decisions (e.g., 5-fluoruracil is less effective in MIN patients) whereas the results of germline testing impact cancer surveillance for the patient and identification of at-risk family members. ${ }^{11,12}$

Clearly, we are entering the era of comprehensive molecular analysis. This includes examining tumors for sequence changes, abnormal methylation, rearrangement (translocations and fusions), and copy number changes. The $A L K$ proto-oncogene is an example of a single gene that undergoes multiple different mechanisms of activation in both the germline and somatically in tumors that range from neuroblastoma to lung cancer. ${ }^{13}$ Increasingly, sophisticated analysis of the tumor genome will improve prediction of inherited susceptibility and will aid in distinguishing which rare constitutional variants are clinically relevant. To speed up the progress, there needs to be better communication among the range of practitioners and researchers working on this problem such that the term "cancer genetics" has one definition. 


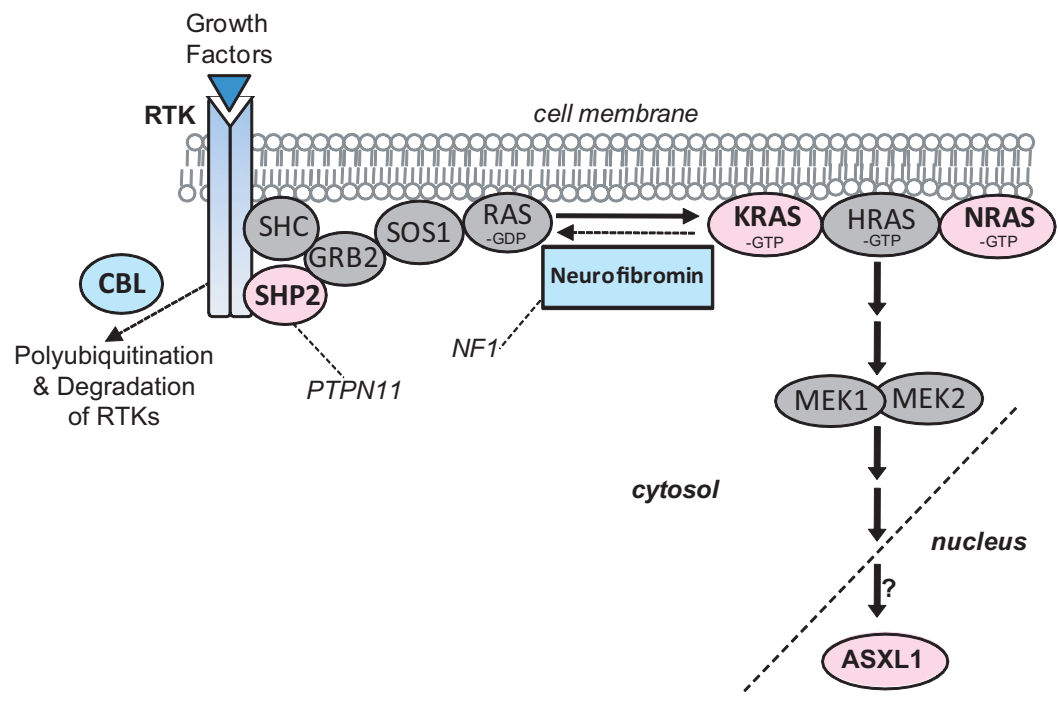

Fig. 1. Schematic diagram of the Ras signaling pathway which is disrupted in juvenile myelomonocytic leukemia (JMML). Inherited susceptibility to JMML genes are depicted in light blue. Genes with somatic mutation in JMML are depicted in pink. The impact of Ras signaling on ASXL1 function is not clear. RTK, receptor tyrosine kinase. Modified with permission from Curr Opin Genet Dev. ${ }^{14}$

\section{ACKNOWLEDGMENTS}

This study was supported by a Grant from the National Cancer Institute 1R01CA138836 (to S.E.P.).

\section{REFERENCES}

1. Rushlow D, Piovesan B, Zhang K, et al. Detection of mosaic RB1 mutations in families with retinoblastoma. Hum Mutat 2009;30:842-851.

2. Cancer Genome Atlas Research Network. Comprehensive genomic characterization defines human glioblastoma genes and core pathways. Nature 2008;455:1061-1068.

3. Armengol C, Cairo S, Fabre M, Buendia MA. Wnt signaling and hepatocarcinogenesis: the hepatoblastoma model. Int J Biochem Cell Biol $2011 ; 43: 265-270$.

4. López-Terrada D, Gunaratne PH, Adesina AM, et al. Histologic subtypes of hepatoblastoma are characterized by differential canonical Wnt and Notch pathway activation in DLK+ precursors. Hum Pathol 2009;40: 783-794.

5. Aretz S, Koch A, Uhlhaas S, et al. Should children at risk for familial adenomatous polyposis be screened for hepatoblastoma and children with apparently sporadic hepatoblastoma be screened for APC germline mutations? Pediatr Blood Cancer 2006;47:811-818.

6. Curia MC, Zuckermann M, De Lellis L, et al. Sporadic childhood hepato- blastomas show activation of beta-catenin, mismatch repair defects and p53 mutations. Mod Pathol 2008;21:7-14.

7. Sugimoto Y, Muramatsu H, Makishima H, et al. Spectrum of molecular defects in juvenile myelomonocytic leukaemia includes ASXL1 mutations. Br J Haematol 2010;150:83-87.

8. Lynch HT, Lynch JF, Lynch PM, Attard T. Hereditary colorectal cancer syndromes: molecular genetics, genetic counseling, diagnosis and management. Fam Cancer 2008;7:27-39.

9. Domingo E, Laiho P, Ollikainen M, et al. BRAF screening as a low-cost effective strategy for simplifying HNPCC genetic testing. $J$ Med Genet 2004;41:664-668.

10. Loughrey MB, Waring PM, Tan A, et al. Incorporation of somatic BRAF mutation testing into an algorithm for the investigation of hereditary nonpolyposis colorectal cancer. Fam Cancer 2007;6:301-310.

11. Ogino S, Nosho K, Kirkner GJ, et al. CpG island methylator phenotype, microsatellite instability, BRAF mutation and clinical outcome in colon cancer. Gut 2009;58:90-96.

12. de la Chapelle A, Hampel H. Clinical relevance of microsatellite instability in colorectal cancer. J Clin Oncol 2010;28:3380-3387.

13. Cheng M, Ott GR. Anaplastic lymphoma kinase as a therapeutic target in anaplastic large cell lymphoma, non-small cell lung cancer and neuroblastoma. Anticancer Agents Med Chem 2010;10:236-249.

14. Tidyman WE, Rauen KA. The RASopathies: developmental syndromes of Ras/MAPK pathway dysregulation. Curr Opin Genet Dev 2009;19: $230-236$. 